\title{
The effect of deep versus moderate neuromuscular block on postoperative respiratory function in bariatric laparascopic surgery: a randomized, double blind clinical study
}

\author{
Baete S, Vander Laenen M, De Vooght P, et al. ZOL Genk, Belgium
}

\section{Introduction}

Laparascopic bariatric surgery poses special demands on both the surgeon and anesthesiologist. The surgeon requires good visualization of the surgical field while the anesthesiologist is concerned with the post-operative respiratory function of these morbidly obese patients. It has been suggested that deep neuromuscular block (NMB) improves surgical conditions during laparoscopy. However, deep NMB puts the patient at risk for residual postoperative NMB and associated respiratory complications. We tested the hypotheses that reversal of deep NMB with sugammadex could prevent postoperative respiratory impairment.

\section{Methods}

In this single centre, randomized, double-blind, clinical trial sixty patients were evenly randomized over a deep NMB-group and a moderate NMB-group. NMB-depth was monitored throughout the procedure by acceleromyography of the adductor pollicis muscle. Anesthesia was induced and maintained with propofol and remifentanil. 15 Minutes prior to extubation a single dose of fentanyl $100 \mu \mathrm{g}$ was administered.

The postoperative pulmonary function was assessed by measuring peak expiratory flow (PEF), forced expiratory volume in 1 second (FEV1) and forced vital capacity (FVC) using an electronic portable peak flow meter. Baseline pulmonary function tests were performed during the preoperative anesthesiology consultation. Data are presented as mean+/-SEM. Additionally the need for postoperative respiratory support and the level of sedation were evaluated. This study was funded by a grant from Merck, Sharp \& Dohme.

\begin{tabular}{|c|c|c|}
\hline \multicolumn{2}{|c|}{74 Patients assessed } & \multirow{2}{*}{$\begin{array}{l}\text { Age > } 18 \text { years } \\
\text { BMI > } 30 \mathrm{~kg} / \mathrm{m}^{2} \\
\text { ASA score < IV }\end{array}$} \\
\hline \multicolumn{2}{|c|}{60 Patients randomized } & \\
\hline \multicolumn{2}{|c|}{ Deep NMB group } & Moderate NMB group \\
\hline \multicolumn{2}{|c|}{ 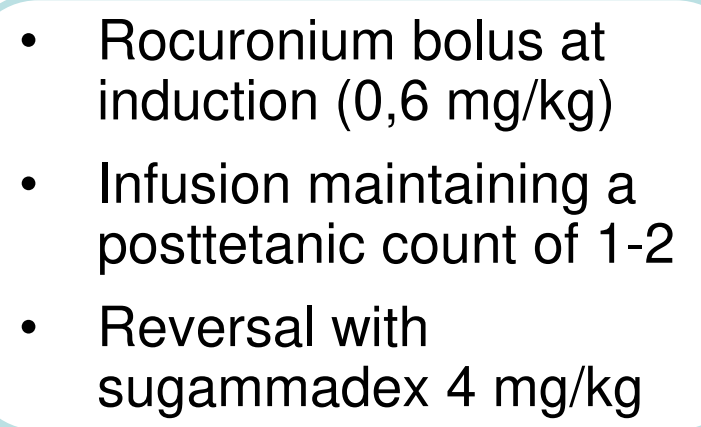 } & 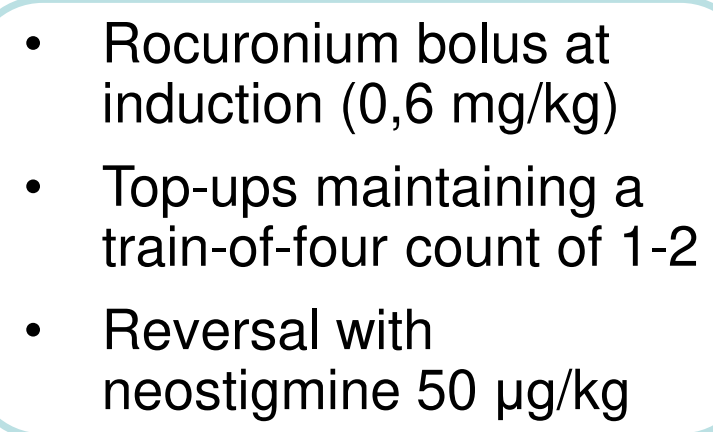 \\
\hline & \multicolumn{2}{|c|}{ Extubation when TOF ratio was $>0.9$} \\
\hline \multicolumn{3}{|c|}{$\begin{array}{l}\text { Evaluation of the need for postoperative respiratory support } \\
\text { and measurement of postoperative pulmonary function in the } \\
\text { recovery room }\end{array}$} \\
\hline
\end{tabular}

\section{Patient characteristics}

11 Patients declined to participate, two were excluded because of prior gastric banding, one patient was excluded because of renal failure. All patients received the allocated medication and there was no loss to follow-up. Baseline characteristics between groups were similar:

\begin{tabular}{ll|l|l} 
Age & & $41 \pm 2$ & $41 \pm 2$ \\
\hline Sex: & Male & $8(27 \%)$ & $4(13 \%)$ \\
& Female & $22(73 \%)$ & $26(87 \%)$ \\
\hline BMI & & $40 \pm 1$ & $41 \pm 1$
\end{tabular}

\section{Results}

After surgery, all the pulmonary function tests were considerably impaired in both groups when compared to baseline. There was no statistically significant difference in the decrease in PEF, FEV1 and FVC (expressed as \% change from baseline) between the deep and the moderate NMB-group.

\section{Relative Post-operative Change}

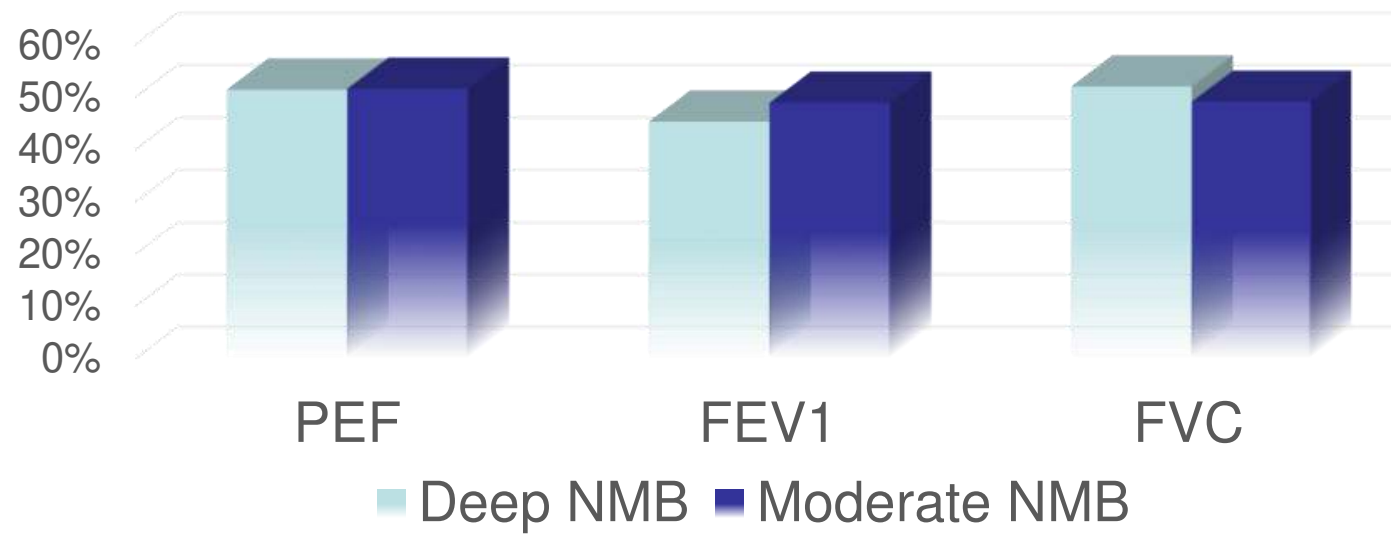

The modified observer's assessment of alertness scale at the time of pulmonary function testing was similar between the 2 groups $(4.9 \pm 0.1$ vs. $4.9 \pm 0.1 ; \mathrm{P}=0.76$ ).

In the deep NMB-group, 2 patients required postoperative non-invasive CPAP vs. 1 patient in the moderate NMB-group $(\mathrm{P}=0.6)$. No patient needed reintubation after surgery.

The following tables compare pre- and post-operative pulmonary function for both groups. A similar decline in post-operative pulmonary function was found in both groups.

\section{Pulmonary Function Tests}

\begin{tabular}{l|c|c|c|}
\multicolumn{5}{c}{ Deep NMB } \\
\cline { 2 - 4 } & Preoperative & Postoperative & P-value \\
\hline PEF (I/min) & $314 \pm 20$ & $141 \pm 15$ & $P<0.0001$ \\
\hline FEV1 (I) & $2.4 \pm 0.2$ & $1.1 \pm 0.1$ & $P<0.0001$ \\
\hline FVC (I) & $3.0 \pm 0.2$ & $1.4 \pm 0.1$ & $P<0.0001$
\end{tabular}

\begin{tabular}{l|c|c|c|}
\multicolumn{4}{c}{ Moderate NMB } \\
\hline & Preoperative & Postoperative & P-value \\
\hline PEF (I/min) & $276 \pm 15$ & $126 \pm 0.1$ & $P<0.0001$ \\
\hline FEV1 (I) & $2.2 \pm 0.1$ & $1.1 \pm 0.1$ & $P<0.0001$ \\
\hline FVC (I) & $2.7 \pm 0.1$ & $1.2 \pm 0.1$ & $P<0.0001$
\end{tabular}

\section{Conclusion}

Pulmonary function was significantly impaired after laparoscopic bariatric surgery in the deep as well as the moderate NMB-group. There was no difference between both groups with respect to the extent of impairment (45 to $52 \%$ decrease from baseline) in PEF, FEV1 and FVC. These results are in line with previous research showing an important decrease in post-operative pulmonary function after laparoscopic surgery, irrespective of the NMB-protocol used, as long as NMB is adequately monitored and reversed at the end of surgery. 